\title{
Analysis and Strategy Research on Information Security in the Era of Big Data
}

\author{
Lei Yao \\ Chengdu Polytechnic, Chengdu, Sichuan, 610041, China \\ 565169780@qq.com
}

Keywords: Data security; Strategy research; Big data

\begin{abstract}
In the era of big data, the information security has attracted much attention. The content of our research can provide guarantee for information security in the big data environment for the construction of a new safe Internet, escort information security, and improve the related hardware security products in the high performance, which is also an inevitable trend of the development of international information security products, with considerable prospects.
\end{abstract}

\section{Introduction}

With the deepening and rapid development of the Internet, the Internet has penetrated into every aspect of our life, and the way of human life is facing tremendous changes. The new Internet also brings all kinds of new ways of life: online shopping, online payment, video chat, e-mail and so on. The convenience of the network brings people new experience, however, various data including some personal privacy data also outbreaks, therefore, the higher requirement is put forward for the data security in the network. If the key data security problems can not be solved efficiently, the phenomenon of privacy leakage and capital loss will seriously restrict the development of the Internet in the fields of electronic commerce and so on, thus affecting the pace of the Internet.

\section{Analysis of Information Security in the Era of Big Data}

In the world of data explosion, data security has been paid more and more attention, which has also become an important index to measure whether the network is mature or not. In the process of the development of the new Internet, IPv4/iPv6 will coexist for a long time, and there will be a variety of transition schemes in this process, which also poses new challenges to network security. If the information security problem can not be solved effectively, the advance of the new Internet will be hindered, and it will also affect the progress of the new Internet industry chain, thus the new technology field and related applications and equipment based on the new Internet will become useless and can not be realized normally.

With the rapid development of "Internet plus", the problem of data security has penetrated into every field of industries and business functions. In the era of big data, data security accidents do not happen by accident. According to the survey, about $2 / 3$ of the data security accidents are caused by human error and system problems, and the global average cost of each damaged data record is as high as \$154. In the next ten years, perhaps longer "big data" times, most enterprises need data security solutions with higher security, larger capacity and faster data storage and recovery to cope with the possible outbreak of data security accidents caused by the explosive growth of data.

In February 2014, the Central Committee set up the central network security and informatization leadership group headed by president $\mathrm{Xi}$, which indicated that the state had already raised information security to the national strategic level. In 2015, the fifteenth meeting of the Standing Committee of the 12th National People's Congress voted on July 1 and passed the new national security new. Information security has become the commanding height of the national strategic security. This project not only ensures information security, but also promotes the development of related industries, and greatly improves the level of China's information security technology, constantly optimizes the structure of the information security industry in China, and flourishes the information security market. The $R \& D$ content of the project belongs to the category of information 
security, which is consistent with the development of the times and will also promote the development of information security technology.

The study of new Internet should fully reflect the controllable, credible and manageable characteristics. A credible new Internet should have the following features: The first one is to realize the confidentiality, integrity and availability of the system and information. The second one is authenticity, that is the authenticity of user identity, source of information, and information content. The third one is the auditability, that is, any action initiated by a network entity can be traced to the entity itself. The fourth one is the privacy, that is, the privacy of users is protected, and some applications are anonymous. The fifth one is invulnerability, which can provide effective service in the environment of system failure and malicious attack. The sixth one is the controllability, which refers to the ability to control violations of network security policies.

On the basis of controllable, trusted and manageable condition, the development of new Internet security guarantee related information security products can achieve the following results in the new Internet security construction and research.

R\&D and industrialization of equipment: Domestic information security equipment has fully occupied the market.

Business Innovation: It brings further prosperity to China's Internet security business.

Technology Innovation: The innovation of network security technology under IPv6, and the accumulation of relevant standard system and intellectual property right.

\section{Analysis of Information Security Technology}

The content of our research can provide a guarantee for information security in the big data environment for building a new secure Internet, and can escort information security. Improving the relevant hardware security products in high performance is also the inevitable trend of the development of international information security products, with considerable prospects.

The new Internet services are divided into the following categories according to the service category: information class, social class, entertainment class, payment class, service class, business class, etc., as shown in Table 1.

Table 1 Examples of new Internet business classification

\begin{tabular}{|c|l|}
\hline $\begin{array}{c}\text { Service } \\
\text { categories }\end{array}$ & \multicolumn{1}{|c|}{ Business examples } \\
\hline $\begin{array}{c}\text { Information } \\
\text { class }\end{array}$ & $\begin{array}{l}\text { Weather information, mobile phone map, news } \\
\text { information, entertainment information, mobile } \\
\text { phone search, etc. }\end{array}$ \\
\hline Social class & Mobile IM, SNS, etc. \\
\hline $\begin{array}{c}\text { Entertainme } \\
\text { nt class }\end{array}$ & $\begin{array}{l}\text { Mobile music, mobile video, mobile games, and } \\
\text { so on }\end{array}$ \\
\hline $\begin{array}{c}\text { Payment } \\
\text { class }\end{array}$ & Mobile payment, NFC, etc. \\
\hline $\begin{array}{c}\text { Service } \\
\text { class }\end{array}$ & LBS, mobile phone reservation, etc. \\
\hline $\begin{array}{c}\text { Business } \\
\text { class }\end{array}$ & $\begin{array}{l}\text { Mobile phone mailbox, mobile office, cloud } \\
\text { storage, mobile phone translation, etc. }\end{array}$ \\
\hline
\end{tabular}

The huge data will inevitably bring some difficult problems. How to complete the integration, query and encryption of big data more effectively is the center of many scholars' research.

The research content of this project includes the comprehensive research on data security technology, including the process of the implementation of related technologies in the entity, which is mainly embodied in the related practical new technologies (see Figure 1). 


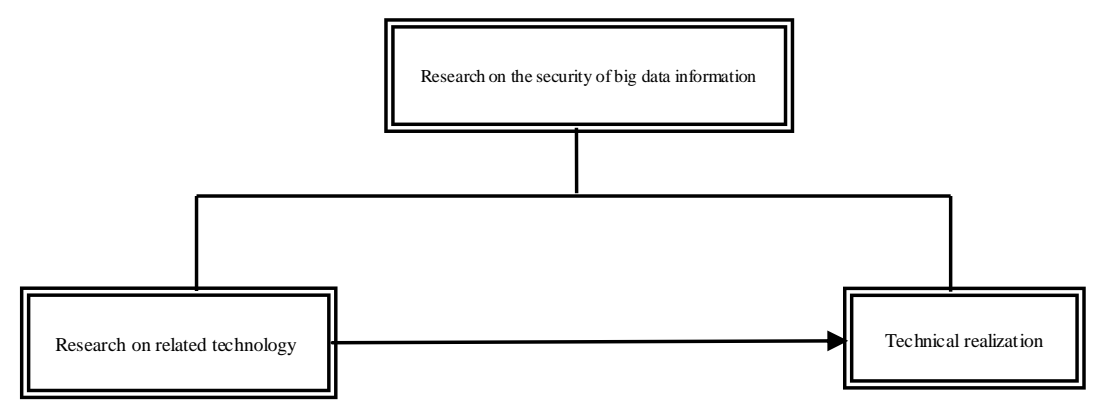

Figure 1. Mission planning for information security technology research in the era of big data

A secure data protection system needs to be supported by a complete set of basic equipment. With the support of software and hardware, data security can be fully guaranteed.

The first is the terminal Infrastructure.

Intelligent terminal facilities have basically integrated all kinds of complex functions such as the Internet and telecommunications service. In order to further meet the demand of new Internet business in the new Internet ear for smart terminals, we also need to break through many key technical issues such as hardware and software for many terminal devices.

The second is the network infrastructure.

The basic network facilities of the new Internet mainly include the wireless access network, the backbone network of the Internet and the mobile core network (packet domain), among which the mobile core network (packet domain) and the wireless access network belong to the mobile communication network.

The third is the application infrastructure.

The new Internet is becoming more and more personalized, differentiated and long tailed, so as to ensure that the application infrastructure can support the mobile Internet applications properly. At the same time, the construction of application infrastructure also helps to capture the market in a timely manner through the deployment of rapid development business, and through reliable certification and authorization to achieve the security management of users and businesses. Combined with a reasonable billing and charging strategy, the maximum return can be obtained.

Big data is bound to be linked to new networks. Healthy and safe network systems include:

The first one is the application Security

The first point is the content filtering

Web content filtering: Web content filtering is based on the URL of the classification library for access control. It can selectively control various negative websites, such as pornography, reactionary, and so on, and filter the Web page keywords and mobile code such as Java, JavaScript, ActiveX, etc.

Anti spam: The address of sending and receiving mail, the attachment name, the contents of the attachment, the main body, the content of the body, the name of the senders and recipients are matched and filtered.

The second point is the application access control.

The application access control provides a unified identity authentication mechanism based on identity tokens and digital certificates and access control based on attribute certificates. It can fully protect the controlled information from illegal and unauthorized access, and provide sufficient reliable basis for subsequent tracking, thus completing subsequent appointment. Business.

The third point is the security audit.

The security audit includes two types of audit strategies: One is that the system audit strategy that controls which events should be recorded as the system related activity, including the subject identification, the change of privileges, and the management of the security policy(such as modifying access control data). The other is to apply audit strategy ti control which events the application should audit.

The second one is the network security. 
The new Internet network mainly consists of two parts, the access network and the IP bearer network / Internet. When access network uses mobile communication network, it involves base station (BTS), base station controller (BSC), wireless network controller (RNC), mobile switching center (MSC), media gateway (MGW), service general packet radio service support node (SGSN), gateway general packet radio service support node (GGSN) and other devices and related links, and when it uses $\mathrm{WiFi}$, it is associated with access (AP) devices. The IP bearer network / Internet mainly involves routers, switches, access servers and other devices and the coordination of related links.

The first point is encryption and authentication.

At present, the encryption and authentication system can refer to the WPKI authentication system. WPKI (WAP Public Key Infrastructure) is generated from the main ideas of the PKI standard, and related improvements are made to the WAP security specification and the particularity of the mobile Internet. The WAP security specification includes three parts: WAP transport layer security specification WTLS, WAP application layer security specification, WIM specification and WAP certificate management specification.

The second point is the network isolation exchange.

The network isolation exchange can achieve the security isolation between two interconnected networks, and completes the exchange of the specified packets between the two networks as required. The system can set two independent network processing units, and each network processing unit only corresponds to one connected network. Each network processing unit only has a unique isolated data channel. As a results, the two network processing units are physically formed to be two independent entities, so as to realize the non-crossing of data exchange, and each network processing unit is completely independent and is no longer affected by other network processing units. The object of processing and exchange between units is no longer the IP data message. It is converted to the encapsulated application layer data message. This message will be encapsulated through the special internal protocol, which realizes the purpose of security exchange.

The third point is the abnormal flow control.

In the environment of address features, sessions number is counted. Traffic management and Diffserv service level are set through policy methods. Abnormal traffic control makes traffic statistics on protocol, address, service port, packet length and so on. At the same time, the maximum / minimum / priority bandwidth control and DSCP service level setting can be carried out, and the two-way flow control can be realized.

Big data volume level real-time protection and emergency recovery technology research and development and industrialization projects are based on virtual disk volume technology, PB level big data volume real-time protection algorithm, volume level IO interception and separation, ESXI virtual machine automatic generation and other technology research and development. The algorithm is implemented on the mirror volume, which does not change the IO operation of the production volume to maximize the safety of the production volume. It solves the problem of big data security systematically and takes over immediately when the production server fails, which is very suitable for big data enterprises such as intelligent manufacturing and cloud platform, and can greatly improve the overall stability and security of the cloud computing infrastructure, big data applications and other fields. It is also the focus of our research on this project.

With the development of society, people's demand for new networks is getting higher and higher, and the development of big data must also keep pace with the times. All kinds of new information security problems are still emerging, therefore, we are bound to keep studying and meet more difficult and severe test with a strong heart.

\section{Acknowledgements}

Research project funded by Chengdu Polytechnic "Analysis of Data Security and Real-Time Protection Technology in Big Data Era"(17CZYG0225). 


\section{References}

[1] Ran Dong: Intelligent Parking Management Application Based on Mobile Internet[J], China Internet, 2012-11-15

[2] Liang Wei: Security Framework of Mobile Internet[J], China Information Security, 2011-07-15

[3] Zhijiang Zhang and Ni Zhang: Security of the Next Generation Internet[J], ZTE Technology Journal, 2011-04-1

[4] Jie Chen, Jianwei Liu, Mengmeng Wang, Shuangyu He and Jian Mao: Security Substrate Based Security Management and Control Mechanism of Reconfigurable Network[J], Telecommunications Science, 2014-07-20

[5] Gongming Wang, Yong Guan, Chunjiang Zhao and Huarui Wu: Trusted Network Framework and Research[J], Computer Engineering and Design, 2007-03-16

[6] Baidu Wenku: Review of Network Research[J], Internet Document Resources, 2012-11-22

[7] Xinkai Jia and Qingfeng Gu: Research on Mobile Internet Security.[J], Mobile Communications, 2011-05-30

[8] New National Security Act: Establishing the "Overall National Security Concept", Law and Life, 2015-07-15

[9] Difan Song and Jianqi Huang: Looking at the Development of Mobile Internet from the Perspective of Operators[J], Mobile Communications, 2011-10-15.

[10] Zhihui Xiao: A Review of Mobile Internet Research[J], Telecommunications Science, 2009-10-15 